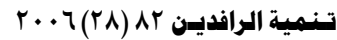

$$
\begin{aligned}
& \text { ص ص[? }
\end{aligned}
$$

$$
\begin{aligned}
& \text { استخدام طريقة الامكان الأعظم وطريقة كابلن - ميلير }
\end{aligned}
$$

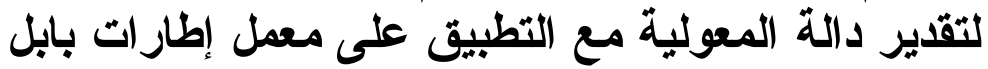

\author{
الاكتور صفاء يونس الصفاوي \\ أستاذ مساعد - قسم الإحصاء

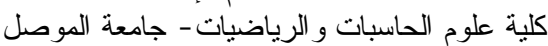

\title{
المستخلص
}

ظهرت دراسة المعولية Reliability في العقد الأول من القرن العشرين ثم ازداد الاهتمام

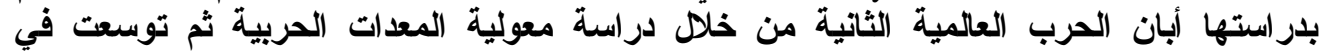

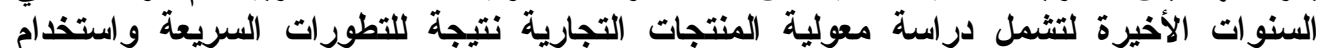

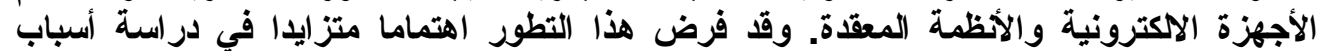

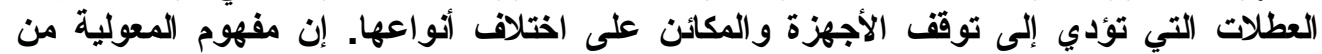

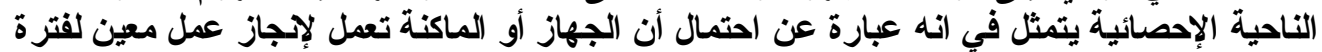
من الزمن حتى حصول العطل في هذه فئه المئه الماكنة.

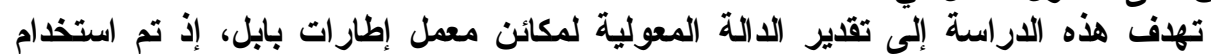

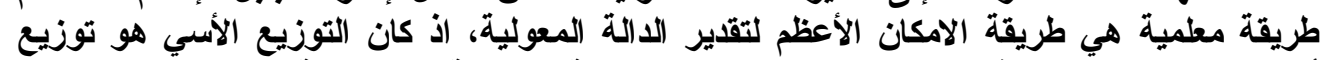

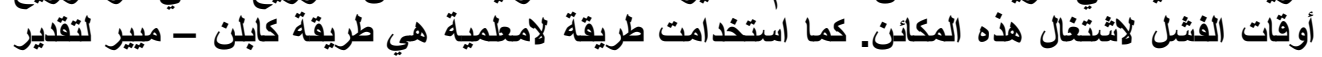

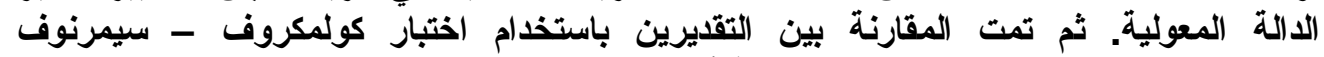
Kolmogorov - Smirnov ومن خلال المقارنة أتضح انه لم يكن هناك فرق معنوي كبير بين استخدام

\section{The Use of Maximum Likelihood and Kaplan-Meir Method to Estimate the Reliability Function An Application on Babylon Tires Factory}

\author{
Dr. Safa'a Y. Saffawy \\ Dept. of Statistics-Mosul University
}

Zakaria Y. Al-Jammal

Dept. of Statistics-Sulaimani University

\section{ABSTRACT}

The study of reliability has appeared in the first decade of the twentieth century. The concentration on this type of study has been crystallized during the (II) World War, via studying the military devices reliability. This type has expanded recently to include the study of commercial products as a result of extraordinary developments on the one hand; and the use of electronic devices and the complex systems on the other. This sort of development has imposed

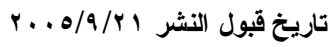

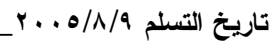


an increasing concern on studying the reasons of breakdowns that may lead to the stoppage of devices and sets in their various kinds.

So, the concept of reliability is statistically the probability that the device and/or set may work to fulfill a certain work for a span of time until the breakdown has occurred.

The current study aims at estimating the reliability function of Babylon Tires Factory. Two methods have been followed (parametric and non-parametric). The first method is the maximum likelihood method as a parametric one. The second is Kaplan-Meir method as a nonparametric. A distribution has been demonstrated throughout using Komogrov - Simirove test. It is concluded that there was no significant difference between the two methods.

\section{Introduction المقدمة}

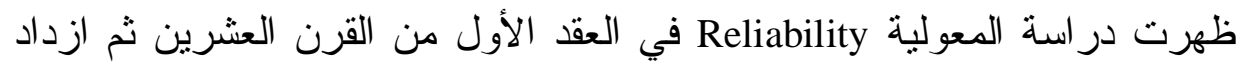
الاهتمام بدر استها أبان الحرب العالمية الثانية من خلال در استة معولية المعدات الحربة الحربية

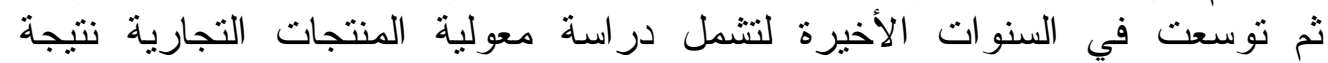

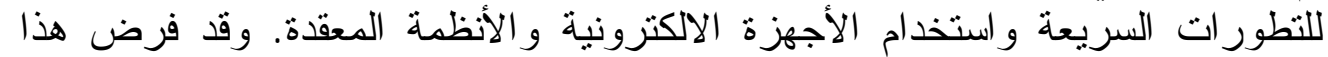

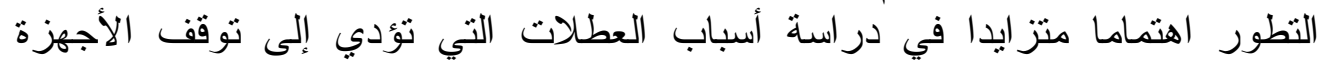

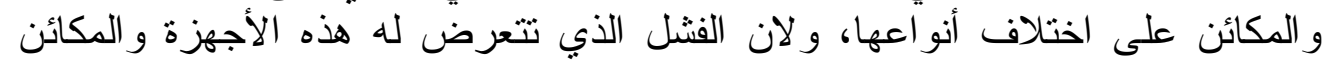

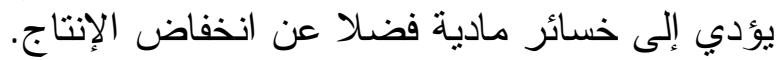

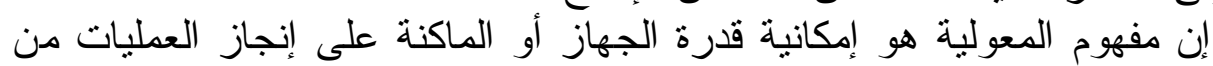

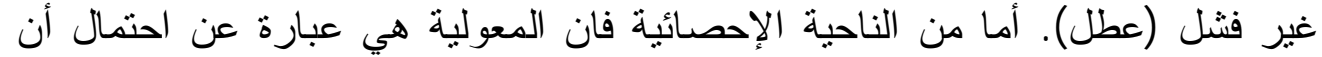

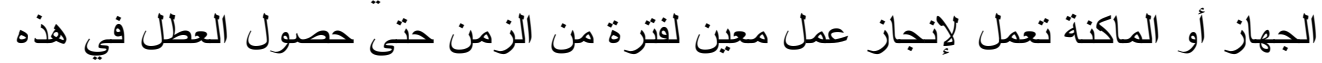

تهدف هذه الدر اسة إلى تقدير دالة المعولية لمكائن معمل إطار ات بابل (حيث تم

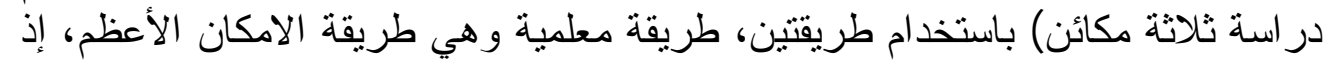

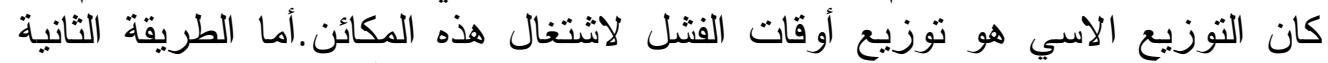

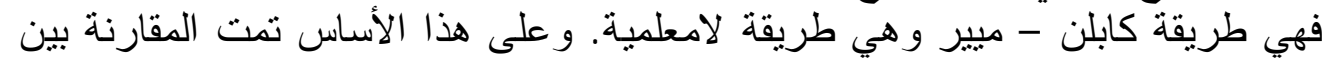

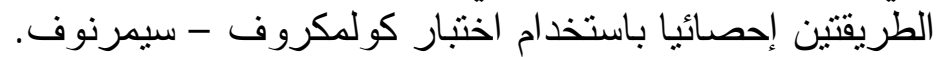

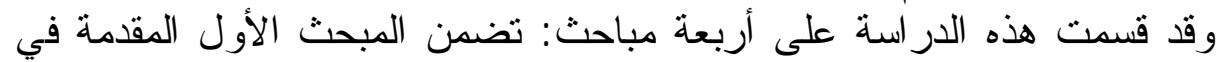

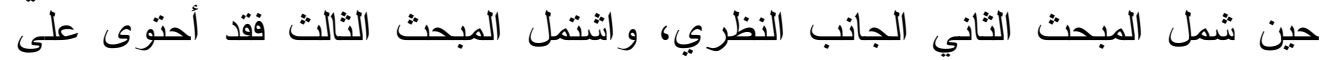
الجانب النطبيقي أما المبحث الر ابع على الاستنتاجات.

\section{r. r. الجاتب النظري: بعض المفاهيم الخاصة بالمعولية}

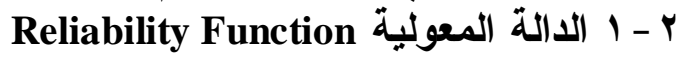

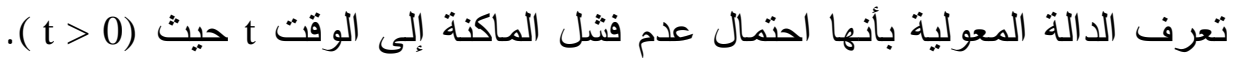

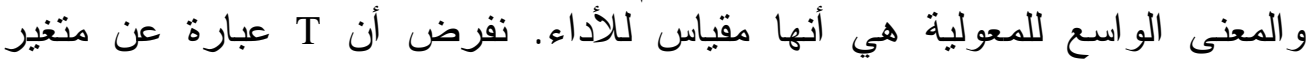




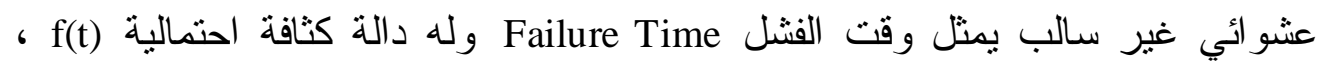

$$
\begin{aligned}
& \text { فضلا عن دالة احتمالية تجميعية F(t) فإن : } \\
& \boldsymbol{R}(t)=p(T>t), \quad 0<t<\infty \\
& R(t)=1-p(T \leq t) \\
& \text { ويمكن إعادة كتابة المعادلة (1) بالشكل الاتي الثة : } \\
& =1-F(t)
\end{aligned}
$$

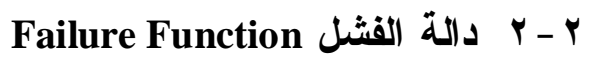

يمكن تعريف دالة الفشل بأنها احتمال فثنل (عطل) الماكنة خلال المدة المدأ بالر بالرمز (t) f(t) . و تعطى صيغة دالة الفشل بالثكل الاتي: $f(t)=\lim _{\Delta t \rightarrow 0} \frac{p_{r}(t<T<t+\Delta t)}{\Delta t}$

(Kalbfleisch and Prentice, 1980,6) (نظر

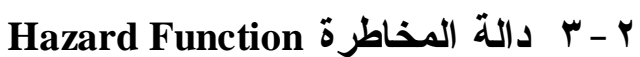

تعرف دالة المخاطرة بأنها المعدل الفوري Instantaneous Rate لحدوث الفئ الفشل

عندما T=t . أما التعريف الرياضي لدالة المخاطرة أو ما بسمى أحيانا بنسبة الفشل

$$
\begin{aligned}
h(t) & =\lim _{\Delta t \rightarrow 0} \frac{p_{r}(t<T<t+\Delta t \mid T>t)}{\Delta t} \\
& =\frac{f(t)}{R(t)}
\end{aligned}
$$

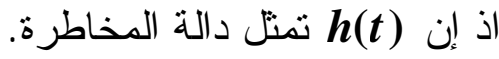

Failure Time Distributions r - بوزيعات أوقات الفشل

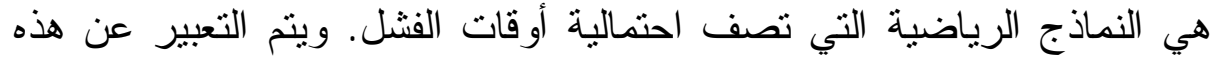

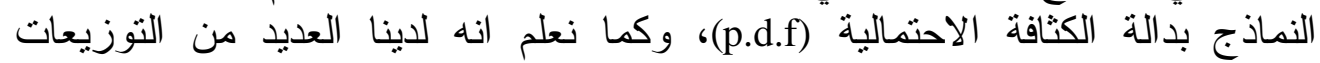

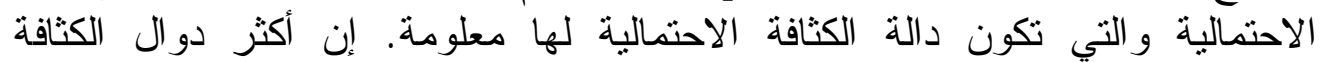
الاحتمالية التي تمثل أوقات الفثل تثبع توزيعات احتمالية معروفة، ومن أكثة الكثر هذه 


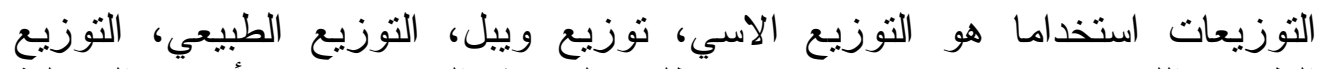

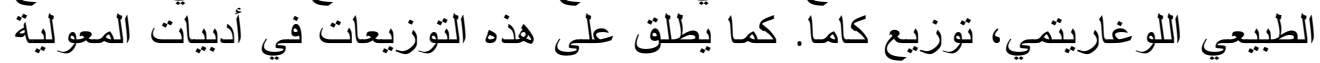

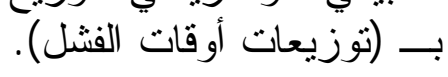

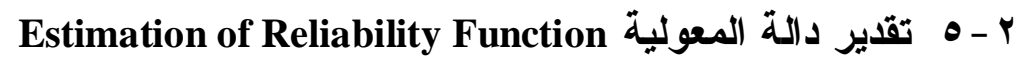

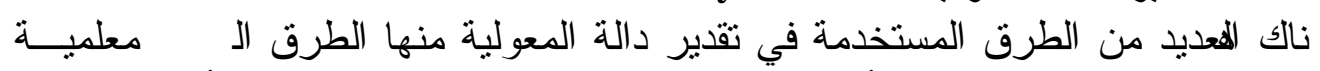

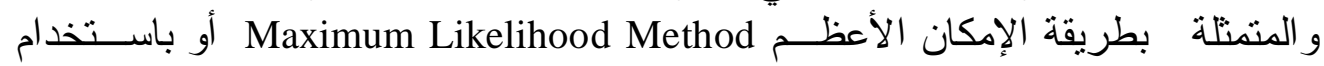

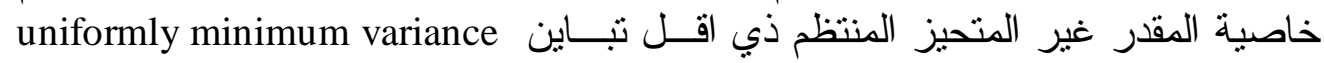
unbiased Estimator

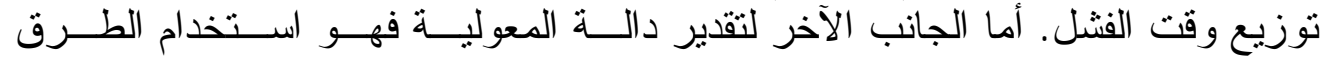

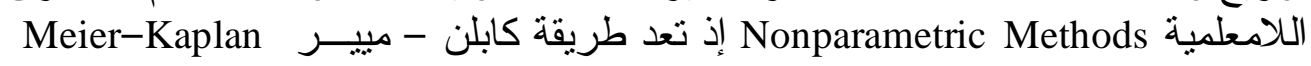

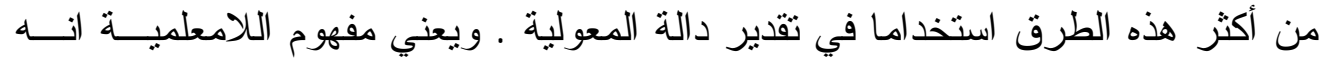
ليس لدينا توزيع معروف (معلوم) للمعلمات.

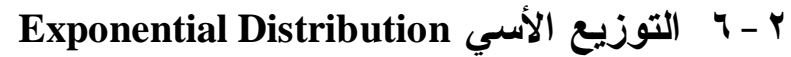

يعد التوزيع الأسي أكثر توزيعات الفشل استخداما في دراسة المعولية. ودالة

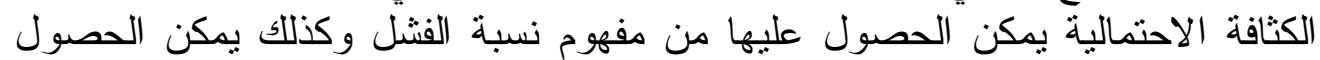

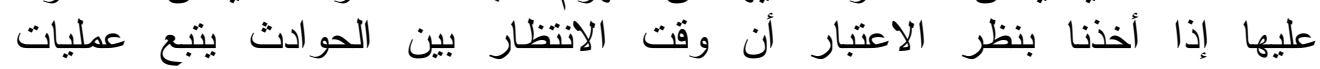

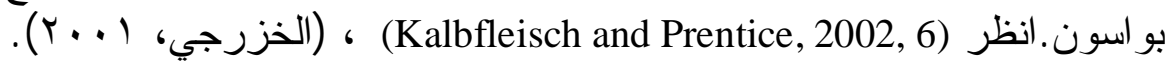

$$
\begin{aligned}
& f(t)=\frac{1}{\theta} \operatorname{EXP}\left(-\frac{t}{\theta}\right), t>0 \\
& \text { إن دالة الكثافة الاحتمالية (p.d.f) هي : } \\
& \text { وان دالة التوزيع التجميعية (c.d.f) هي : } \\
& F(t)=1-E X P\left(-\frac{t}{\theta}\right) \\
& R(t)=E X P\left(-\frac{t}{\theta}\right)
\end{aligned}
$$




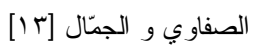

$$
\begin{aligned}
h(t) & =\frac{f(t)}{R(t)} \\
& =\frac{1}{\theta}
\end{aligned}
$$

ولغرض تقدير معلمة القياس $\theta$ الخاصة بالتوزيع الأسي فإننا وكما سبق سنستخدم التقدير بطريقة الإمكان الأعظم و على النحير النحو الآتي:

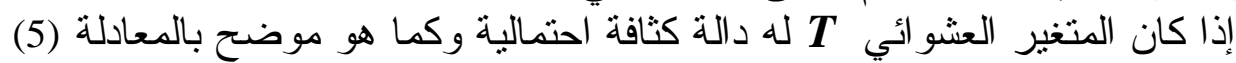
ومن المعروف أن دالة الامكان هي الفئي :

$$
\begin{aligned}
& L(\theta)=\prod_{i=1}^{n} f\left(t_{i} ; \theta\right) \\
& L(\theta)=\prod_{i=1}^{n}\left[\frac{1}{\theta} \operatorname{EXP} \quad\left(-\frac{t_{i}}{\theta}\right)\right] \\
& =\theta^{-n} \operatorname{EXP}\left(-\frac{\sum_{i=1}^{n} t_{i}}{\theta}\right)
\end{aligned}
$$

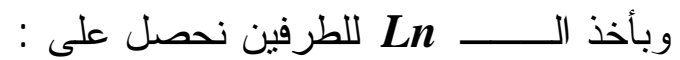

$$
\begin{aligned}
& \operatorname{Ln} L(\theta)=-n \operatorname{Ln} \theta-\frac{\sum_{i=1}^{n} t_{i}}{\theta}
\end{aligned}
$$

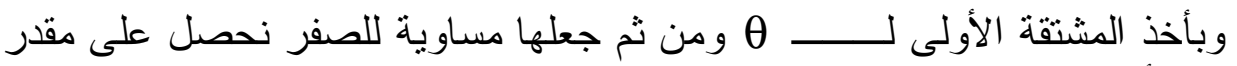
الامكان الأعظم (MLE) وبالثكل الألئي

$$
\hat{\theta}=\frac{\sum_{i=1}^{n} t_{i}}{n}=\bar{t}
$$

و عليه يكون التقدير 
الآن وبعد أن قدرت ف فوف يتم تقدير دالة المعولية وذلك من خلال تعويض قيمة مقدر الامكان الأعظم فر في دالة المعولية الموضحة بالمعادلة 7 و وعلى النحو

$$
\hat{R}\left(t_{i}\right)_{M L E}=\operatorname{EXP}\left(-\frac{t}{\bar{t}}\right)
$$

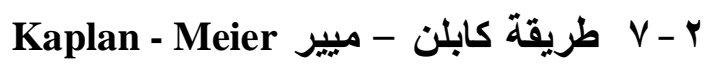

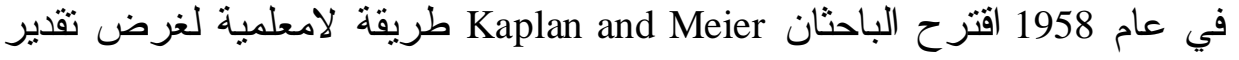

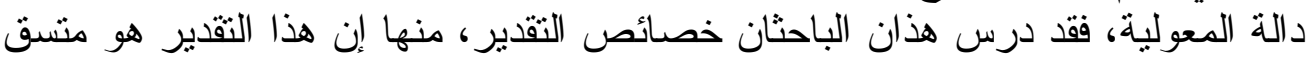
Consistent أن دالة تقدير المعولية باستخدام طريقة كابلن - ميير تعطى بالثكل الآتي:

$$
\hat{R}\left(t_{i}\right)=\prod_{j=1}^{i}\left(\frac{n_{j}-r_{j}}{n_{j}}\right), i=1,2, \ldots \ldots . ., m
$$
: m : أن: : هو العدد الكلي للفتر ات. . : $\boldsymbol{n}_{\boldsymbol{j}}$ • عد مرات الفشل للزمن

$$
n_{j}=n-\sum_{j=0}^{i-1} S_{j}-\sum_{j=0}^{i-1} r_{j} \quad, i=1,2, \ldots \ldots \ldots m
$$
n : العدد الكلي للوحدات تحت التحليل.

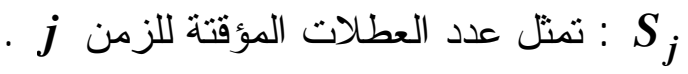

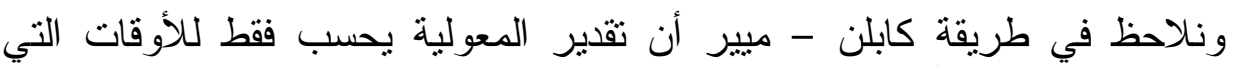
يظهر فيها فثل واحد أو اكثر. في حين بسنتبد عدد العطلات المؤقتة عن عملية التشغيل وتضاف إلى المجموع n

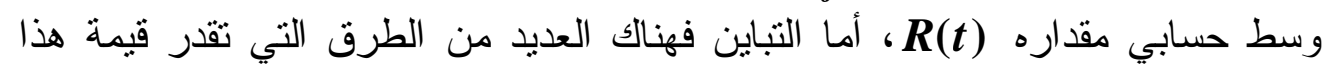
التباين، ومن أكثر الصيخ استخداما هي صيغة Greenwood (Grenwood, 1926) . 


$$
\begin{aligned}
& \operatorname{Var}[\hat{\mathbf{R}}(\mathbf{t})]=[\hat{\mathbf{R}}(\mathbf{t})]^{2} \sum_{j=1}^{\mathbf{t}(\mathbf{j}) \leq t} \frac{\mathbf{r}_{\mathbf{j}}}{\mathbf{n}_{\mathbf{j}}\left(\mathbf{n}_{\mathbf{j}}-\mathbf{r}_{\mathbf{j}}\right)} \\
& \text { وهناك طريقة أخرى، قدمها الباحث (Peto, 1977) و هي: } \\
& \operatorname{Var}[\hat{R}(t)]=[\hat{R}(t)]^{2}\left[\frac{1-\hat{R}(t)}{n_{k}}\right]
\end{aligned}
$$

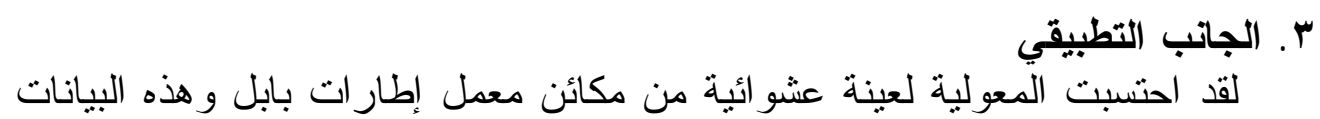

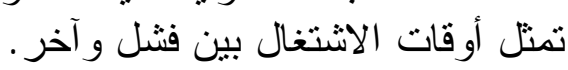
r - 1 وصف البيانات

لقد تم الاعتماد على بيانات لعينة عشو ائية لمكائن معمل إطار ات باتبل بابل تم تناولها

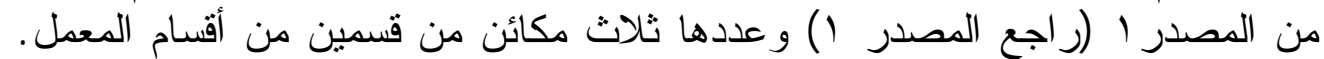

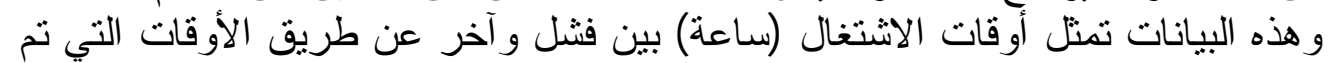

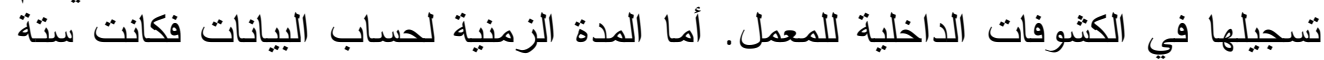

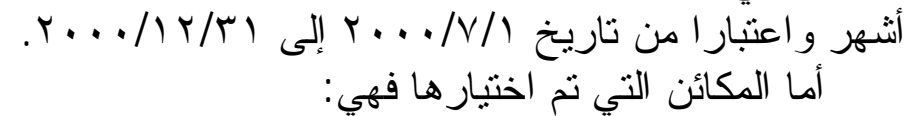

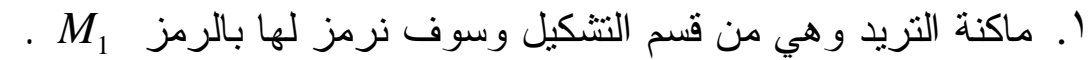

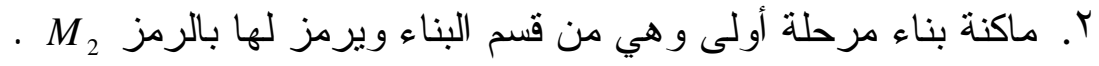

\begin{tabular}{|c|c|}
\hline \multicolumn{2}{|l|}{ أوقات الاشتغال (ساعة) بين فشل وآخر للمكائن الثلاث } \\
\hline أوقات الاشتغال بين فثل و آخر & رمز الماكنة: \\
\hline $\begin{array}{l}18.75,4,259.5,19,203.5,24,261,96,321,402.5,404,72,127.5,10.5, \\
135,247,17,2.5,292.5,8.5,19.25,152,11.5,83.25,17.5,147,44.5,66.5, \\
245\end{array}$ & $M_{1}$ \\
\hline $\begin{array}{l}140.5,312,22.5,48.75,72.5,49.75,218.25,22.25,9.25,68.25,0.25,75.5, \\
22.5,23.25,22,63,23,58.75,237.5,193.5,30.25,17.75,141,146.5,127.5 \text {, } \\
257.75,352,42.5,138.5,51,35.75,173,41.75\end{array}$ & $M_{2}$ \\
\hline $\begin{array}{l}71.25,141.75,78,220.25,80.25,44,752.25,268,200.5,194,14.5,11.75, \\
2.5,90.75,43,49.75,13,84.75,140.75,38.25,77,26.75,51.25,78.5,34.5, \\
53.5,118.75,5,33,19.5,18.75,47,15.5,31.75,60,11.5,9,130.75\end{array}$ & $M_{3}$ \\
\hline
\end{tabular}

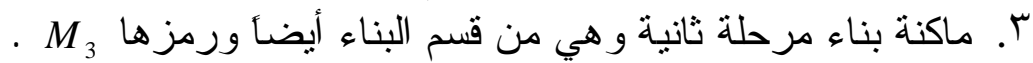
الجدول ا يوضح أوقات الاشتغال بين فنثل و آخر للمكائن الثثلاث وباء الثنكل الاتي: 


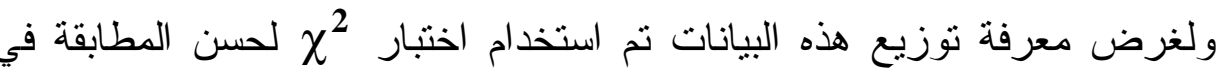

برنامج MINITAB ولقد تبين بان أوقات الاشتغال بين فنثل و آخر لها توزيع أسي.

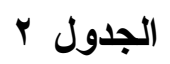

تقدير دالة المعولية لـ

\begin{tabular}{|c|c|c|c|c|}
\hline No. & time & $\mathbf{r j}$ & R(ti)MLE & $\mathbf{R}(\mathbf{t i}) \mathbf{K M}$ \\
\hline 1 & 2.5 & 1 & 0.98066 & 0.9655 \\
\hline 2 & 4 & 1 & 0.969235 & 0.931 \\
\hline 3 & 8.5 & 1 & 0.935755 & 0.8966 \\
\hline 4 & 10.5 & 1 & 0.921248 & 0.8621 \\
\hline 5 & 11.5 & 1 & 0.91408 & 0.8276 \\
\hline 6 & 17 & 1 & 0.875637 & 0.7931 \\
\hline 7 & 17.5 & 1 & 0.872224 & 0.7586 \\
\hline 8 & 18.75 & 1 & 0.863748 & 0.7241 \\
\hline 9 & 19 & 1 & 0.862063 & 0.6897 \\
\hline 10 & 19.25 & 1 & 0.860381 & 0.6552 \\
\hline 11 & 24 & 1 & 0.82904 & 0.6207 \\
\hline 12 & 44.5 & 1 & 0.706359 & 0.5862 \\
\hline 13 & 66.5 & 1 & 0.594821 & 0.5517 \\
\hline 14 & 72 & 1 & 0.569805 & 0.5172 \\
\hline 15 & 83.25 & 1 & 0.521866 & 0.4828 \\
\hline 16 & 96 & 1 & 0.472391 & 0.4483 \\
\hline 17 & 127.5 & 1 & 0.369345 & 0.4138 \\
\hline 18 & 135 & 1 & 0.348327 & 0.3793 \\
\hline 19 & 147 & 1 & 0.317158 & 0.3448 \\
\hline 20 & 152 & 1 & 0.305008 & 0.3103 \\
\hline 21 & 203.5 & 1 & 0.20398 & 0.2759 \\
\hline 22 & 245 & 1 & 0.1475 & 0.2414 \\
\hline 23 & 247 & 1 & 0.145213 & 0.2069 \\
\hline 24 & 259.5 & 1 & 0.131704 & 0.1724 \\
\hline 25 & 261 & 1 & 0.130169 & 0.1379 \\
\hline 26 & 292.5 & 1 & 0.101775 & 0.1034 \\
\hline 27 & 321 & 1 & 0.081461 & 0.069 \\
\hline 28 & 402.5 & 1 & 0.043097 & 0.0345 \\
\hline 29 & 404 & 1 & 0.042595 & 0 \\
\hline
\end{tabular}




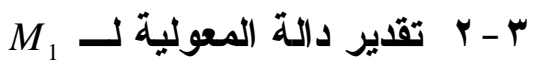

سنقوم بتقدير دالة المعولية للماكنة الأولى باستخدام طريقة الامكان الأعظم المتمثلة بالمعادلة 9 اذ إن بالمعادلة ال ا ـ الجدول ب يوضح قيم دالة المعولية المقدرة لكل وقت بالطريقتين.

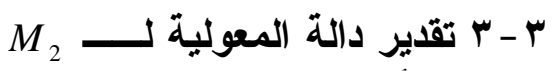

هنا أيضا قمنا بتقدير دالة المعولية للماكنة الثانية باستخدام طريقة الامكان الأعظم

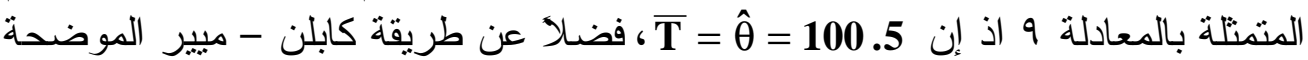

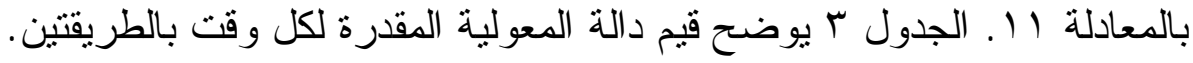

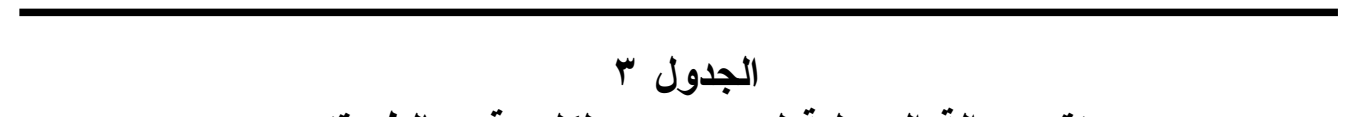

تقدير دالة المعولية لـــــ

\begin{tabular}{|c|c|c|c|c|}
\hline No. & Time & rj & R(ti)MLE & R(ti)KM \\
\hline 1 & 0.25 & 1 & 0.997516 & 0.9688 \\
\hline 2 & 9.25 & 1 & 0.912069 & 0.9375 \\
\hline 3 & 17.75 & 1 & 0.838101 & 0.9063 \\
\hline 4 & 22 & 1 & 0.803398 & 0.875 \\
\hline 5 & 22.25 & 1 & 0.801402 & 0.8438 \\
\hline 6 & 22.5 & 2 & 0.799411 & 0.8125 \\
\hline 7 & 23 & 1 & 0.795443 & 0.7813 \\
\hline 8 & 23.25 & 1 & 0.793467 & 0.75 \\
\hline 9 & 30.25 & 1 & 0.740081 & 0.7188 \\
\hline 10 & 35.75 & 1 & 0.700668 & 0.6875 \\
\hline 11 & 41.75 & 1 & 0.660061 & 0.6563 \\
\hline 12 & 42.5 & 1 & 0.655154 & 0.625 \\
\hline 13 & 48.75 & 1 & 0.615651 & 0.5938 \\
\hline 14 & 49.75 & 1 & 0.609556 & 0.5625 \\
\hline 15 & 51 & 1 & 0.602021 & 0.5313 \\
\hline 16 & 58.75 & 1 & 0.557342 & 0.5 \\
\hline 17 & 63 & 1 & 0.534264 & 0.4688 \\
\hline 18 & 68.25 & 1 & 0.507071 & 0.4375 \\
\hline 19 & 72.5 & 1 & 0.486075 & 0.4063 \\
\hline 20 & 75.5 & 1 & 0.471779 & 0.375 \\
\hline 21 & 127.5 & 1 & 0.281209 & 0.3438 \\
\hline & & & & \\
\hline
\end{tabular}

بتبع 


\begin{tabular}{|c|c|c|c|c|}
\hline No. & Time & rj & R(ti)MLE & R(ti)KM \\
\hline 22 & 138.5 & 1 & 0.252055 & 0.3125 \\
\hline 23 & 140.5 & 1 & 0.247088 & 0.2813 \\
\hline 24 & 141 & 1 & 0.245862 & 0.25 \\
\hline 25 & 146.5 & 1 & 0.232768 & 0.2188 \\
\hline 26 & 173 & 1 & 0.178817 & 0.1875 \\
\hline 27 & 193.5 & 1 & 0.145821 & 0.1563 \\
\hline 28 & 218.25 & 1 & 0.11399 & 0.125 \\
\hline 29 & 237.5 & 1 & 0.09412 & 0.0938 \\
\hline 30 & 257.75 & 1 & 0.076944 & 0.0625 \\
\hline 31 & 312 & 1 & 0.044848 & 0.0313 \\
\hline 32 & 352 & 1 & 0.030122 & 0 \\
\hline
\end{tabular}

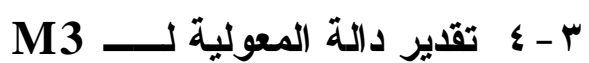

بالأسلوب نفسه الذي اتبعناه في الجدولين السابقين قمنا بتقدير دالة المعولية للماكنة

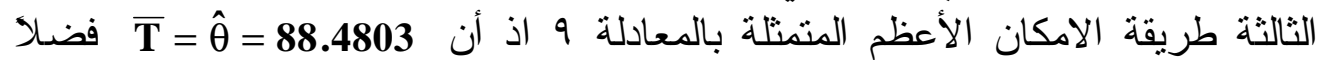

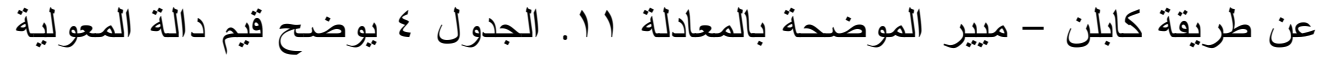
المقدرة لكل وقت بالطريقتين.

\begin{tabular}{|c|c|c|c|c|}
\hline \multicolumn{5}{|c|}{ الجدول ع } \\
\hline No. & Time & $\mathbf{r j}$ & R(ti)MLE & $\mathbf{R}(\mathbf{t i}) \mathbf{K M}$ \\
\hline 1 & 2.5 & 1 & 0.972147 & 0.9737 \\
\hline 2 & 5 & 1 & 0.945069 & 0.9474 \\
\hline 3 & 9 & 1 & 0.903305 & 0.9211 \\
\hline 4 & 11.5 & 1 & 0.878145 & 0.8947 \\
\hline 5 & 11.75 & 1 & 0.875668 & 0.8684 \\
\hline 6 & 13 & 1 & 0.863387 & 0.8421 \\
\hline 7 & 14.5 & 1 & 0.848876 & 0.8158 \\
\hline 8 & 15.5 & 1 & 0.839338 & 0.7895 \\
\hline 9 & 18.75 & 1 & 0.809074 & 0.7632 \\
\hline 10 & 19.5 & 1 & 0.802247 & 0.7368 \\
\hline 11 & 26.75 & 1 & 0.739146 & 0.7105 \\
\hline
\end{tabular}




\begin{tabular}{|c|c|c|c|c|}
\hline No. & Time & rj & R(ti)MLE & R(ti)KM \\
\hline 12 & 31.75 & 1 & 0.698544 & 0.6842 \\
\hline 13 & 33 & 1 & 0.688747 & 0.6579 \\
\hline 14 & 34.5 & 1 & 0.677172 & 0.6316 \\
\hline 15 & 38.25 & 1 & 0.649077 & 0.6053 \\
\hline 16 & 43 & 1 & 0.615158 & 0.5789 \\
\hline 17 & 44 & 1 & 0.608246 & 0.5526 \\
\hline 18 & 47 & 1 & 0.587973 & 0.5263 \\
\hline 19 & 49.75 & 1 & 0.569984 & 0.5 \\
\hline 20 & 51.25 & 1 & 0.560405 & 0.4737 \\
\hline 21 & 53.5 & 1 & 0.546337 & 0.4474 \\
\hline 22 & 60 & 1 & 0.507648 & 0.4211 \\
\hline 23 & 71.25 & 1 & 0.44705 & 0.3947 \\
\hline 24 & 77 & 1 & 0.418928 & 0.3684 \\
\hline 25 & 78 & 1 & 0.414221 & 0.3421 \\
\hline 26 & 78.5 & 1 & 0.411887 & 0.3158 \\
\hline 27 & 80.25 & 1 & 0.403823 & 0.2895 \\
\hline 28 & 84.75 & 1 & 0.383803 & 0.2632 \\
\hline 29 & 90.75 & 1 & 0.358644 & 0.2368 \\
\hline 30 & 118.75 & 1 & 0.261373 & 0.2105 \\
\hline 31 & 131.75 & 1 & 0.225666 & 0.1842 \\
\hline 32 & 140.75 & 1 & 0.203845 & 0.1579 \\
\hline 33 & 141.75 & 1 & 0.201555 & 0.1316 \\
\hline 34 & 194 & 1 & 0.111683 & 0.1053 \\
\hline 35 & 200.5 & 1 & 0.103774 & 0.0789 \\
\hline 36 & 220.25 & 1 & 0.083018 & 0.0526 \\
\hline 37 & 268 & 1 & 0.0484 & 0.0263 \\
\hline 38 & 752.25 & 1 & 0.000203 & 0 \\
\hline
\end{tabular}

؛ ـ الاستنتاجات

ا. من خلال الجدول r نـاحظ بأنه لايوجد فرق معنوي كبير في تقدير معولية الماكنة

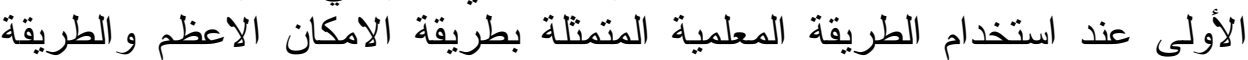

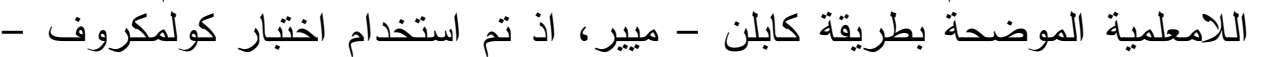

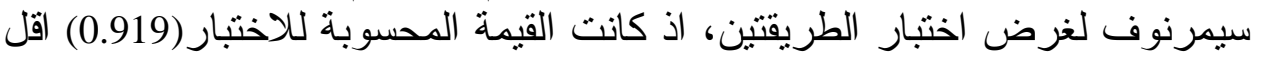

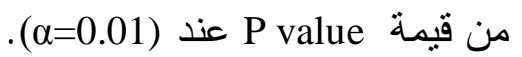


r. عند تقدير دالة معولية الماكنة الثانية أيضا لم نلحظ وجود فرق معنوي كبير بين

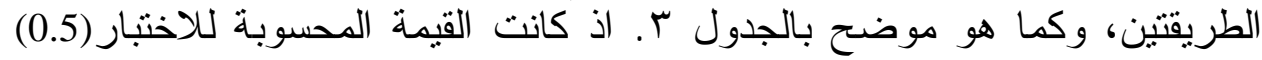

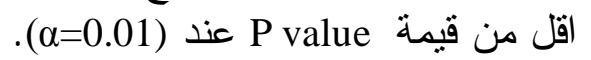

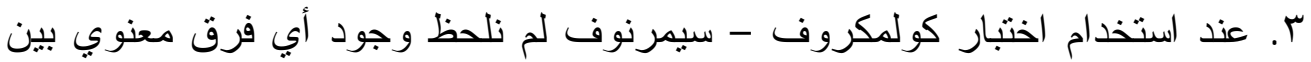

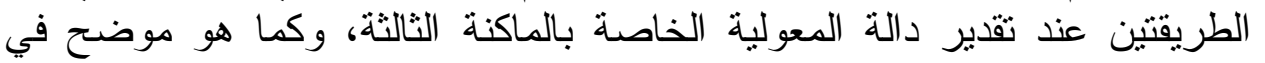
الجدول ع. اذ كانت القيمة المحسوبة للاختبار (0.574) اقل من قيمة . $(\alpha=0.01)$

ع. يتبين من النقاط الثلاث المذكورة آنفأ أنه بالامكان الاعتماد على الطريقة اللامعلمية

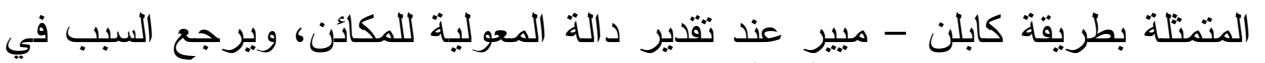

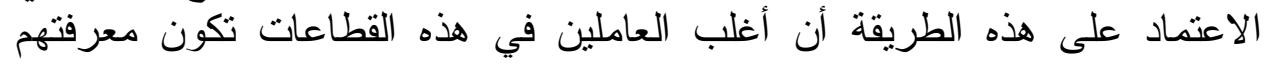

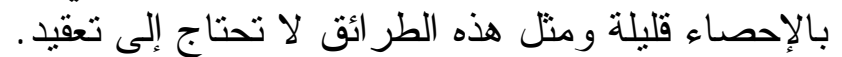

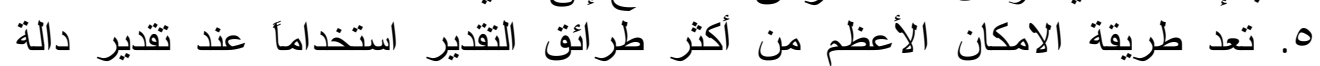
المعولية، وذلك لسهولة استخدام هذه الطريقة في إيجاد مقدر ات معلمات لاتفات توزيعات

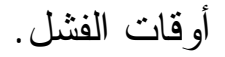

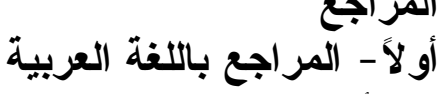

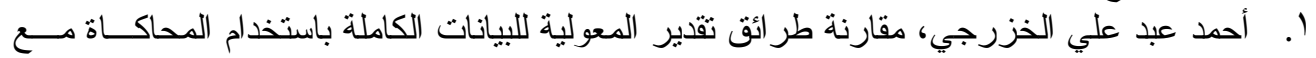

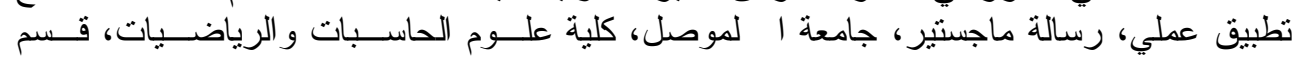

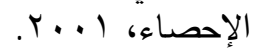

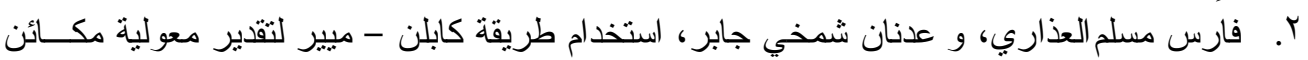
قسم المربيات/معمل تعليب كربلاء، مجلة تنمية الر افدين، العدد العشرون، I9NV

\section{ثانياً - المراجع باللغة الاجنبية}

1. Ebeling, C.E.,An Introduction to Reliability and Maitamability Engineering, McGraw-Hill, 1997.

2. Greenwood, M., The Errors of Sampling of Survivorship tables, Reports on Public Health and Statistical Subjects, 1926.

3. Kalbfleisch, J.D. and Prentice, R.L., $2^{\text {nd }}$ ed., The Statistical Analysis of Failure Time Data, John Wiley and Sons. New York, 2002.

4. Kaplan, E.L, and Meier, P., Nonparametric Estimation From Incomplete Observations, JASA, 1958.

5. Lawless, Life Time Distribution, Estimation and Testing, John Wiley and Sons, New York, 2003.

6. Peto, R. et al, Design and Analysis of Randomized Clinical Trials Requiring Prolonged Observation of each Patient, British Journal of Cancer, 1977. 